\section{Original} Article

\title{
Pressure Controlled Clamp Using Shape Memory Alloy for Minimal Vessel Invasion in Blood Flow Occlusion
}

\author{
Ye Zhang, MD, ${ }^{1}$ Hiroyasu Kanetaka, PhD, ${ }^{1,2}$ Yuya Sano, DDS, ${ }^{2}$ Mitsuhiro Kano, PhD, ${ }^{3}$ \\ Tadaaki Kudo, $\mathrm{PhD},{ }^{4}$ Takumi Sato, DDS, ${ }^{5}$ and Yoshinaka Shimizu, $\mathrm{PhD}^{5}$
}

\begin{abstract}
Purpose: Vessel damage after clamping may affect the success of surgical operations. A new pressure controlled clamp (SMA clamp) was designed using super elastic property of shape memory alloy (SMA) to realize atraumatic vessel occlusion. The ability and biological effect of the SMA clamp to control pressure was investigated in vivo.

Methods: The loading-displacement curves of the SMA clamps (experimental group) and conventional clamp (control group) by occlusion of pig carotid arteries were evaluated using a clamping-pressure analyzing system. To investigate macroscopically and histologically the vessel damage of the SMA and conventional clamps, pig carotid arteries were stained with Evan's blue and its histological sections were stained with Elastica Massion after clamping for fifteen minutes.

Results: Constant value was shown in the loading-displacement curve of SMA clamp. In the control group, damaged area stained with Evan's blue in the vessel wall showed enlargement with the pressure increasing. Less areas in experimental groups are observed than that in the control group. Histological section in the experimental group showed no obvious except a slight compressive damage in the tunica intima. In the control group, vessel wall showed irreversible damages.

Conclusions: This experiment indicated that the SMA clamp, which has a unique mechanical property, can be used without vessels damage. This pressure controlled clamp can be a selection in clinical apparatus to improve surgical safety.
\end{abstract}

Keywords: clamp, vessel damage, shape memory alloy, pressure control

\section{Introduction}

Clamps to arrest flow and to control bleeding from arteries have been an important instrument in surgical op- erations since 400 years ago. ${ }^{1,2)}$ However, high pressure of clamps cause moderate to severe damage to the vessel wall, leading to unsuccessful surgical operation. Peri-operative complications caused by clamps, such as arterial
${ }^{1}$ Department of Physical Medicine and Rehabilitation, Graduate School of Biomedical Engineering, Tohoku University, Sendai, Miyagi, Japan

${ }^{2}$ Division of Advanced Prosthetic Dentistry, Tohoku University, Sendai, Miyagi, Japan

${ }^{3}$ Division of Oral and Craniofacial Anatomy, Tohoku University, Sendai, Miyagi, Japan

${ }^{4}$ Division of Oral Physiology, and Tohoku University, Sendai, Miyagi, Japan

${ }^{5}$ Division of Oral Pathology, Graduate school of Dentistry, Tohoku University, Sendai, Miyagi, Japan.
Received: December 19, 2011; Accepted: February 7, 2012 Corresponding author: Ye Zhang, MD. Department of Physical Medicine and Rehabilitation, Graduate School of Biomedical Engineering, Tohoku University, 4-1 Seiryo-machi, Aoba-ku, Sendai 980-8575, Japan

Email: ye.zhang@bme.tohoku.ac.jp

(C)2012 The Editorial Committee of Annals of Thoracic and Cardiovascular Surgery. All rights reserved. 
dissection, ${ }^{3)}$ thrombosis ${ }^{4)}$ and creation of an intimal flap ${ }^{5)}$ was reported.

The minimal invasive instrument for vessel has been expected for surgeon. Guthrie and Carrel first introduced the concept of the atraumatic clamp in 1903. ${ }^{6}$ Since then, numerous leaders in the surgical field have subsequently designed atraumatic clamps. Although these clamps have been widely used, they could not fully avoid the vessel damage. Intimal tears and flaps were confirmed in 26 percent of cases using clamps by arteriographic and ultrasonic evaluation. ${ }^{7)}$

It has already been proved that vessel damage arises from the high clamp pressure. Dujovny and Slayback demonstrated that endothelial damage was proportional to the occlusive pressure. ${ }^{6)}$ They introduced a concept of minimal occlusive force (MOF) ${ }^{8,9)}$ which refers to the minimal force to occludes the vessel. They proved that minimal occlusive force minimized damages of vessels. After that, in this respect, much research has been done. These studies proved that the effective control of clamping pressure can improve the safety of the operation. For example, Gregory et al. used a soft angled bulldog clamp to reduce pressure on the vessel wall. ${ }^{10)}$ Ozalp et al. reported an endoluminal balloon technique as an alternative occlusion strategy. ${ }^{11)}$ Darçin et al. reported an atraumatic occluding clamp technique using PTCA balloon. ${ }^{4)}$ This technique decreased the pressure and prevented the damage of the vessel wall. However, because of complication or imprecision, such as extra monitor needed, this technique could not be practically used. ${ }^{12)}$

Clamp structure and material are important factors to control the pressure on the vessel. The basic structure of the conventional clamp occurs exponential increase of pressure with the displacement of the handle. ${ }^{13)}$ Use of conventional clamp made of usual material is difficult to realize minimal vessel invasive technique. ${ }^{8}$ Selecting a new material which facilitates pressure controlling may be a method helpful to realize minimal vessel invasive.

Shape memory alloy (SMA) is an excellent biomaterial that has been widely used in the medical field. ${ }^{14,15)}$ Especially NiTi SMA alloy, which have excellent mechanical properties such as shape memory, springback, and super elasticity is used widely for clinical devices. Gall et al. studied tensile deformation of NiTi wire and proved this material is of benefit as an axial force and elastic foundation using in biomedical devices. ${ }^{16)}$ Luo et al. checked the mechanical property of NiTi wire and proposed taking advantage of this prosperity to realize pressure controlling. ${ }^{2)}$ Zhao designed a laparoscopy using this property in

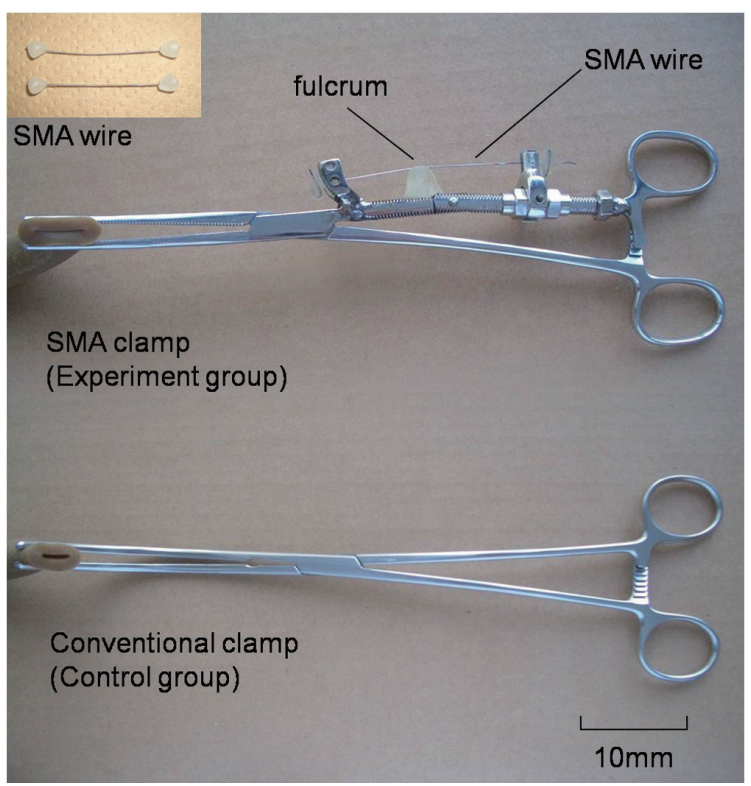

Fig. 1 The newly designed pressure controlled clamp, conventional clamp and SMA wires $(0.4 \mathrm{~mm}$ and $0.5 \mathrm{~mm}$ of diameter). The SMA wire was embedded in one handling branch of the clamp with an additional fulcrum. The wire and fulcrum formed a pressure control structure.

SMA: shape memory alloy

order to improve the safety of minimal invasive operation. ${ }^{17)}$ However, the effect of clamp using super elastic SMA on the in vivo vessel reaction has not been investigated.

The purpose of this study was to histologically investigate the effects of the newly designed SMA clamp to control pressure to reduce the damage on animal carotid vessel. We expected that the properties of the SMA clamps may inhibit the vessel damages and protect the vessels wall in surgical operation.

\section{Materials and Methods}

\section{Design of pressure controlled clamp using SMA}

Pressure controlled clamp (experimental group) was manufactured by improvement of the conventional clamp (Satinsky type, Izumi Co. Ltd, Sendai, Japan) using NiTi shape memory alloy (Ni, 56wt\%; Ti, 44wt\%) (Furukawa Techno Material Co Ltd, Kanagawa, Japan). This clamp can control the pressure in clamp tips by alteration of the diameters $(0.4 \mathrm{~mm}$ and $0.5 \mathrm{~mm})$ of SMA wires (length: $70 \mathrm{~mm}$ ), using of its super elastic property (Fig. 1). The both ends of SMA wire were fixed in a handling branch of the clamp through an additional fulcrum (Fig. 1). The 


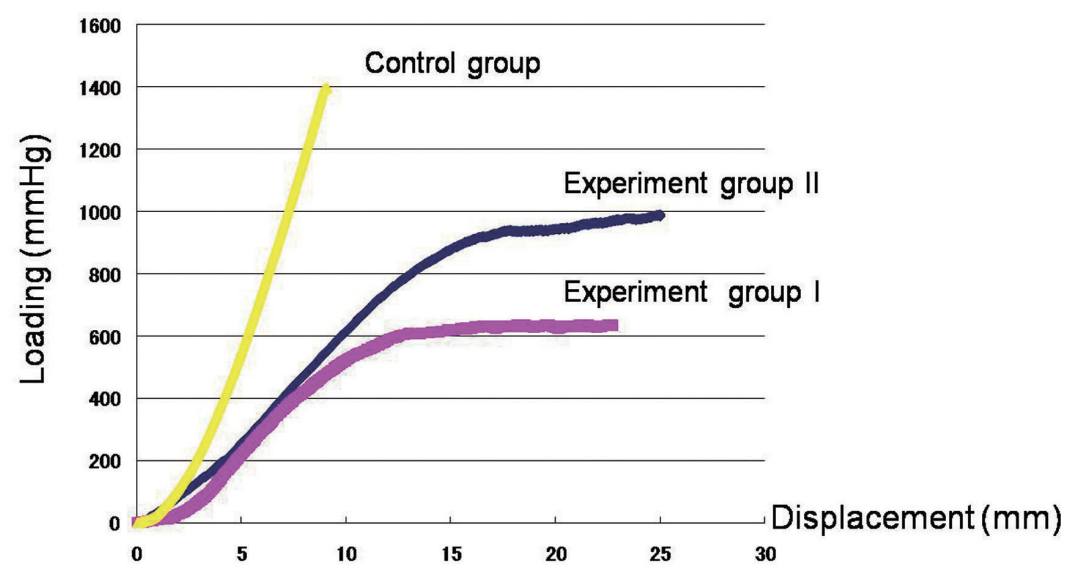

Fig. 2 The loading-displacement reaction of pressure controlled SMA clamps (experimental groups) and the conventional clamp (control group). The diameter of SMA wires used was $0.4 \mathrm{~mm}$ and $0.5 \mathrm{~mm}$, respectively.

SMA: shape memory alloy

SMA wire provides the pressure in clamp tips by elongation of SMA wire during clamp closure. With elongation of the SMA wire, the loading increases to a critical point. After reaching the yield point, a loading saturation is revealed, and the pressure remains constant. Further elongation of the wire shows no increase in the loading. In the control group, a conventional clamp (Satinsky type, Izumi Co. Ltd, Sendai, Japan) was used (dimensions: 65 $\mathrm{mm}$ in length of jaws and $220 \mathrm{~mm}$ in total length).

\section{Mechanical characteristic of pressure controlled SMA clamps}

The clamping-pressure analyzing system was used to investigate the mechanical characteristic of clamps. The experimental setup consists of a holder for fixing one branch of the clamps, and a uniaxal moving stage. The clamp was fixed on the operation stage. An electronic pressure sensor (Kyowa, PSM-2KA, Tokyo) was attached to the jaws of the clamp, $1 \mathrm{~cm}$ from the clamp tips. Its upper handle was fixed to the load cell (Minebea, CB7-3K-11, 29.6 N, Fujisawa). The load cell was moved by a uniaxal moving stage controller (QT-CD1, Chou Precision Industrial Co. Ltd., Tokyo). The pressure sensor and the controller were connected to a computer. After the vessel model had settled between the clamp tips, the load cell was moved vertically downwards. Data of the displacement of load cell and the pressure of the clamp tips were simultaneously collected and analyzed by QT-EDIT software (Kyowa PCD Inc, Tokyo). The velocity was 60 $\mathrm{mm} / \mathrm{min}^{2}{ }^{2}$ For each kind of clamp, the loading-releasing experiment was repeated 3 times.

\section{Vessel preparation}

Thirty healthy male landrace pigs with an average weight of $39.5 \pm 3.7 \mathrm{~kg}$ were used. After intramuscular administration of ketamine chloride $(10 \mathrm{mg} / \mathrm{kg})$ and atropine sulfate $(0.02 \mathrm{mg} / \mathrm{kg})$, anesthesia was induced via an endotracheal tube and maintained with isofluorane, $5 \%$ and 1 to $2 \%$, respectively. The 30 carotid arteries were exposed via a midline neck incision. An aliquot of $150 \mathrm{IU} / \mathrm{kg}$ of sodium Heparin was administered intravenously $5 \mathrm{~min}$ before the first set of clamps was applied to the carotid arteries. Arteries were randomly divided into experimental groups (SMA clamps: experimental group I; SMA wire diameter $0.4 \mathrm{~mm}$, experimental group II; SMA wire diameter $0.5 \mathrm{~mm}$ ) and a control group (conventional clamp). All procedures were approved by the Animal Ethics Screening Committee of the Tohoku University. The animals used in this study received humane care in compliance with the Guide for the Care and Use of Laboratory Animals, published by the Tohoku University, Japan.

\section{Vessel clamping experiment}

The clamping experiment was conducted according to Margovsky and Neville's protocol. ${ }^{9,18)}$ Clamps were placed on the middle section of each artery at $1 \mathrm{~cm}$ intervals, avoiding any branches and surrounding tissues. The clamps were closed to notch I, II, III and IV, and held in place for 15 mins at each notch, perpendicular to the long axis of the vessel and starting from the distal end to avoid clamping a collapsed vessel. The clamps were then removed, and the arteries were checked with Evan's blue stain or histological examination. 


\section{5. in vivo damage evaluation}

Twelve carotid vessels were stained with Evan's blue, for in vivo damage evaluation. After remove of clamps, a $0.5 \%$ solution of Evan's blue dye was administered intravenously and allowed to circulate for $30 \mathrm{~min}$. After sacrifice, the carotid arteries were removed. Carotid diameter was measured, opened longitudinally and pinned flat. The areas stained in blue were photographed by a dissecting stereo microscope at $8 \times$ magnification and measured using digitally captured images with software (NIH image, National Institutes of Health, Bethesda, Maryland).

\section{Elastica masson stain for histological observation}

Eighteen carotid arteries were used for histological observation. Branches and surrounding tissues were removed. The arteries were perfused with $4^{\circ} \mathrm{C}$ phosphate buffer and cut into the 1-cm distance, each corresponding to the site of application of the clamps. Cut arteries were preserved in $10 \%$ formaldehyde, embedded in paraffin and sliced $(6 \mu \mathrm{m})$. After deparaffination, they were transferred to $75 \%$ ethanol for $5 \mathrm{~min}$. After rinsed twice in distilled water, they were left in $3 \%$ potassium dichromate overnight. Then rinsed twice in distilled water and stained with Resorcin Fuchsin for 1 hour and washed in $1 \% \mathrm{HCl}$-alcohol. After rinsing, they were stained with hematoxylin for $5 \mathrm{~min}$, and then moved into ponceau fuchsin for $30 \mathrm{~min}$ and washed with acetic acid. After staining with orange-G for $15 \mathrm{~min}$ and light green for 5 min, they were washed with acetic acid. The slides were then transferred to xylene. The sections were dried and mounted with lemosol. ${ }^{19)}$

\section{Graded analysis of vessel damage}

Damage evaluation of the vessel was quantitatively assessed by graded analysis of the degree of vessel damage using the histological sections with Elastica masson stain. Arteries were histologically observed under microscopy (Leica DM 5000B, Wetzlar, Germany), and determined the grade of vessel damage according to the method described by Moore's et al. ${ }^{20)}$ : grade 0: No injury; grade 1: endothelial deformation, intact endothelium; grade 2: endothelial compressed obviously; grade 3: endothelial tear less than 3mm; grade 4: endothelial denudation, flap greater than $3 \mathrm{~mm}$; grade 5: endothelial and minor medial injury; grade 6: major media injury.

\section{Statistical analysis}

All data are presented as means \pm standard deviation.
Statistical differences were analyzed by two-way ANOVA and Tukey's test using JSTAT 12.5 software. Differences at $p<0.05$ were considered to be statistically significant.

\section{Result}

\section{Mechanical characteristic of pressure controlled clamps}

Figure 2 shows the loading-displacement curve in the control group (conventional clamp) and in experimental groups (pressure controlled clamps). In the control group, the pressure showed a rapid increase with small displacement of handle. The load was over $1400 \mathrm{mmHg}$ at the displacement arrived $10 \mathrm{~mm}$. In the experimental group, load was increased with strain increased until displacement $15 \mathrm{~mm}$. Then the loading showed a constant value with a subsequent increase in displacement. The constant value, was about $600 \mathrm{mmHg}$ in experiment group I and was about $950 \mathrm{mmHg}$ in experiment group II, respectively.

\section{2. in vivo damage evaluation of the vessel wall}

The damaged areas stained with Evan's blue in carotid arteries were shown in Fig. 3. The areas stained with Evan's blue after clamping were different between the control and SMA groups. In the control group, the areas stained with Evan's blue in the carotid arteries was significantly extended with an increase of notch (notch I: $24.53 \pm 17.70 \mathrm{~mm}^{2}$, notch II: $550.66 \pm 241.59 \mathrm{~mm}^{2}$, notch III: $1658.31 \pm 560.07 \mathrm{~mm}^{2}$, notch IV: $969.91 \pm 286.67$ $\mathrm{mm}^{2}$ ). On the contrary, in experimental groups, the area stained with Evan's blue showed no significant change among notch I, II, III and IV (Table 1).

\section{Histological observations of the vessel wall}

The histological findings of vessel stained with Elastica Masson were showed on Fig. 4. Normal carotid artery wall was composed of the tunica intima, media and adventitia. Tunica media of the carotid vessel contained abundant elastic fibers. In the control group, at notch I and II, the tunica intima showed disruption after clamping for fifteen minutes. The tunica media revealed irreversible destruction. At notch III and notch IV, vessel wall were crushed and revealed the separation between tunica media and adventitia. The tunica media were broken with shreds. In experimental groups, the vessel wall showed slightly compressed change at notch I, II, III and IV. 

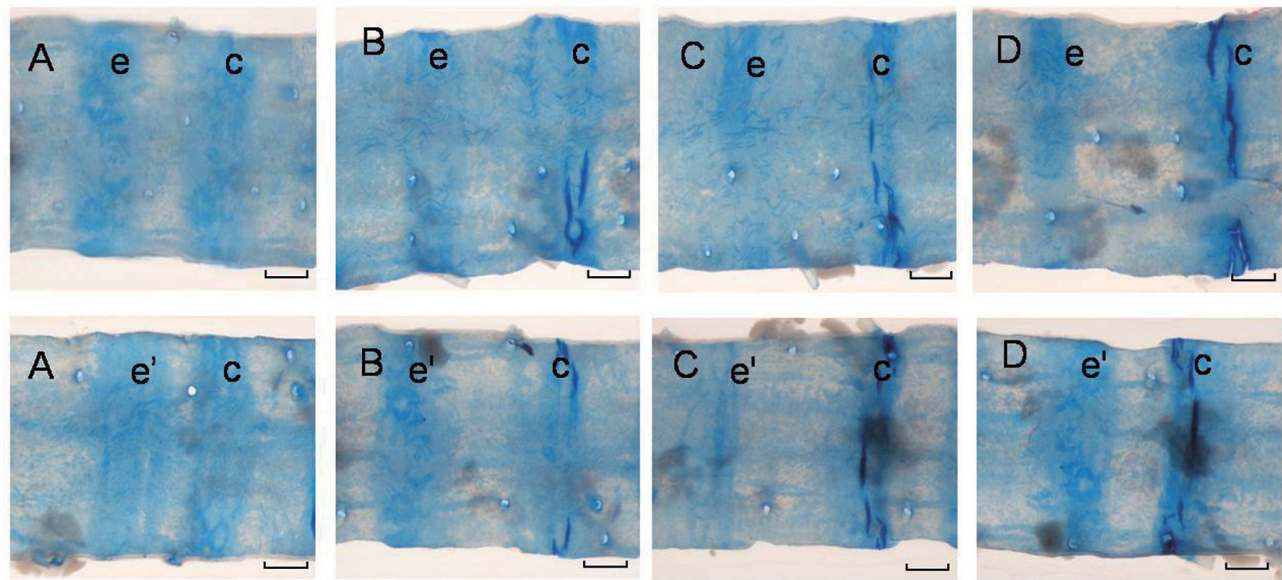

Fig. 3 The representative Evan's blue stain outcome of blood vessels after clamping by pressure controlled clamps (experimental group I, $0.4 \mathrm{~mm}$ SMA wire used; experimental group II, $0.5 \mathrm{~mm}$ SMA wire used) and the conventional clamp (control group) at notches I, II , III and IV respectively. A:notch I; B: notch II; C: notch III; D, notch IV. e: experiment group I; e': experiment group II; c: control group. (stereomicroscope, $\times 8$ )

Table 1 The areas of Evan's blue stain in the vessel wall of carotid arteries seen after clamping by pressure controlled clamps, and the conventional clamp at notches I, II, III and IV respectively

\begin{tabular}{lcccc}
\hline \multirow{2}{*}{ Groups } & \multicolumn{4}{c}{ Trauma area $\left(\mathrm{mm}^{2}\right)$} \\
\cline { 2 - 5 } & Notch I & Notch II & Notch III & Notch IV \\
\hline SMA $(0.4 \mathrm{~mm})$ & $0.53(0.31)$ & $0.92 \pm 0.35$ & $1.16 \pm 0.68$ & $2.86 \pm 0.74$ \\
SMA $(0.5 \mathrm{~mm})$ & $0.77 \pm 0.37$ & $0.47 \pm 0.27$ & $4.69 \pm 0.93$ & $4.87 \pm 1.92$ \\
Conventional & $24.53 \pm 17.70$ & $550.66 \pm 241.59$ & $1658.31 \pm 560.07$ & $969.91 \pm 286.67$ \\
$P$ value $\dagger$ & .0007 & $<.0001$ & $<.0001$ & $<.0001$ \\
\hline
\end{tabular}

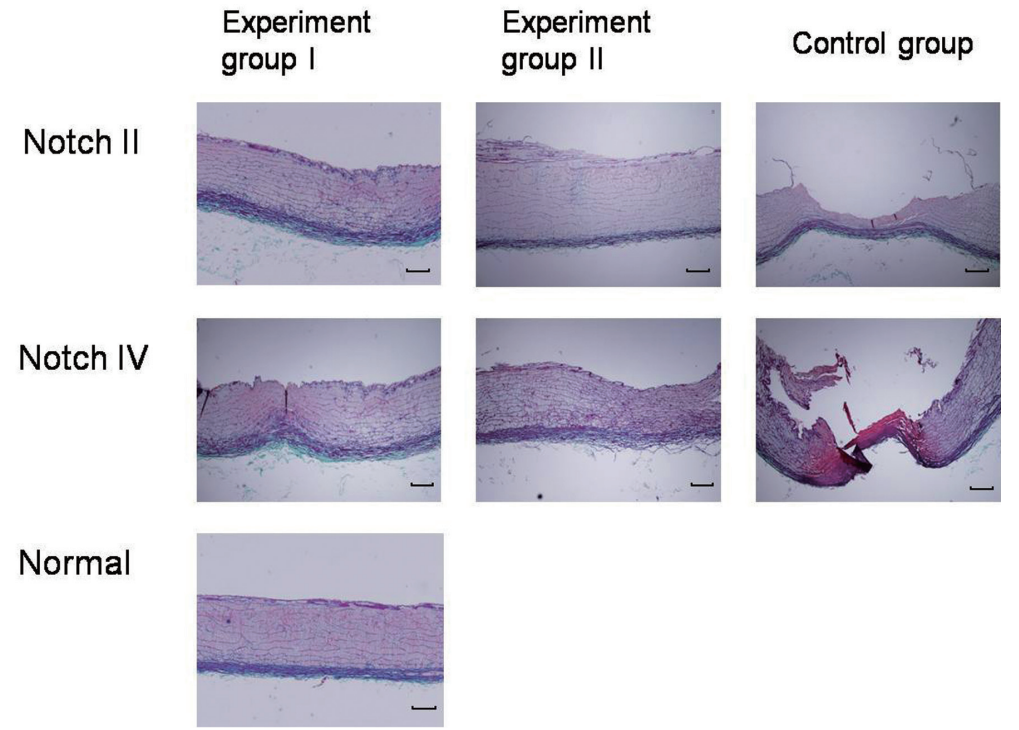

Fig. 4 The representative histological outcome of blood vessels after clamping by pressure controlled SMA clamps (experimental groups) or the conventional clamp (control group), respectively. First column: experiment group, using $0.4 \mathrm{~mm}$ SMA wire used; Second column: experiment group II, 0.5 mm SMA wire used; Third column: control group; Upper row: vessel traverse observation after clamping at notch II; Middle row: vessels traverse observation after clamping at notch IV; Bottom row: normal vessel not be clamped. (elastica masson stains, $\times 200$ )

SMA: shape memory alloy 


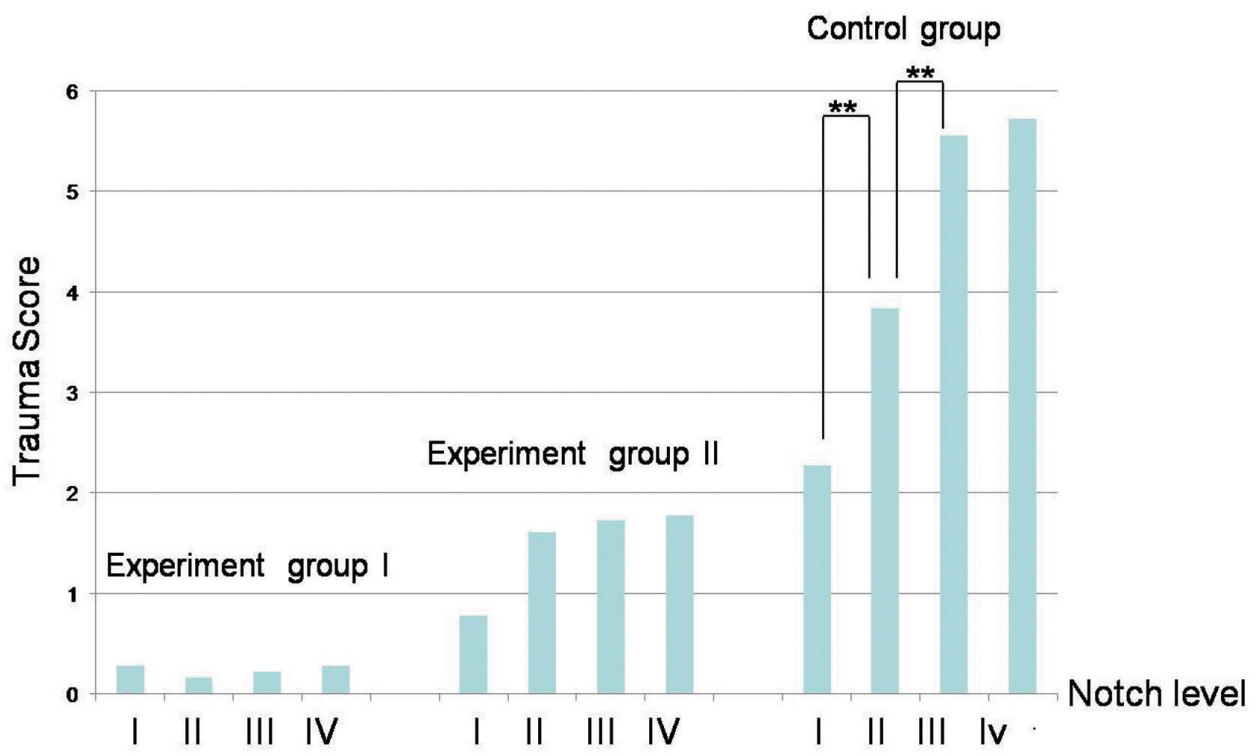

Fig. 5 The damage scores on the vessel wall produced by pressure controlled SMA clamps and the conventional clamp. The diameter of SMA wires used was $0.4 \mathrm{~mm}$ and $0.5 \mathrm{~mm}$, respectively.(**: $p<0.01)$

\section{Graded analysis of vessel damage}

The damage grade of vessels after clamping are illustrated in Fig. 5. It showed that the damage grade of the vessel was correlated with the notch level of clamp. In the control group, damage grade was significant increased with notch level increased ( $3.83 \pm 1.10$ at Notch II to 5.56 \pm 0.51 at Notch III, $5.72 \pm 0.46$ at Notch IV). In the experimental groups, the damage grade showed no significant increase among for notch level (Notch II is $0.38 \pm$ 0.17 (SMA group I) and $0.43 \pm 0.22$ (SMA group II); Notch III is $1.61 \pm 0.50$ ( 0.4 mm SMA group) and $1.72 \pm$ 0.46 (0.5 mm SMA group)).

\section{Discussion}

The special mechanical property of SMA material has been used in medical devices, such as vessel stents ${ }^{14)}$ and orthodontic appliances. ${ }^{15)}$ Because the unique property of super-elasticity that keeps force magnitude constant during deformation is not like other metal material, NiTi SMA is a useful biomedical material, in circumstances that require the control of precise pressure. ${ }^{16)}$ In this study, a new type of pressure controlled clamp using SMA material was designed and investigated in vivo. The basic concept of this new clamp in this study is to ensure that the clamping pressure remains within a safe range through an additional limiter made of the super elastic wire of SMA.
The mechanical properties of the conventional and SMA clamps were examined to understand the pressure control ability. The loading-displacement curve of SMA clamp revealed the constant values of loading over certain handle displacement, unlike loading-displacement curves of conventional clamps. The maintenance of constant value in pressure is important for the practical usage of clamps, because excessive pressure that is higher than minimal occlusive force is the direct reason leading to trauma of vessels. ${ }^{89}$ Constant value of pressure in this new clamp can control by the coordination of the diameter of SMA wire or the alloy elements. This pressure controlled clamp can prepare depending to vessel diameter and individuality.

As a surgical operation device, in vivo reaction of the clamp should be understand before practical use. Pig vessel model was used to evaluate the protective effect in real surgical operation, because the carotid arteries in pig are similar to those in man. We investigated the vessel damage caused by clamp using with Evan's blue staining. Evan's blue dye forms a complex with circulating albumin which stains areas of endothelial disruption or loss. ${ }^{9)}$ The area stained in blue indicates areas in where the dye binds to subendothelial connective tissue. Vessel wall using conventional clamp revealed the gradual extension of blue area with an increase of notch level. In the vessel wall using SMA clamp, no obvious extension of blue area could be observed. These results consisted with findings 
in the histological examination. Combined with the mechanical result, it was proved that after the pressure was controlled, vessel wall damage could be prevented.

After clamping by SMA clamp the thickness of the vessel wall was restored. Conversely, the use of a conventional clamp induced the irreversible damage of the vessel wall. The carotid artery was composed of three tunica, intima, media and advetitia, ${ }^{21)}$ and contains the abundant elastic fibers in tunica media. So though the exceed pressure by clamping will bring the traumatic damage of the vessel wall, optimal pressure may be restored vessel wall to pre-cramping state. Pabst et al. observed that the canine artery caused the intimal damage by elastic vessel loops and Fogarty clamps. $\left.{ }^{6}\right)$ He proved that the vessel wall after clamping revealed the reversible and irreversible changes depending on pressure magnitude. Intimal injuries were minimized when vessel loops are applied lower pressure close to minimal occlusive force (MOF). Our study also represented the same results. Dobrin et al. reported after clamping with DeBakey, Julian-Potts or Fogarty softjaw clamps, chronic histological changes including the intimal hyperplasia and fracture of the internal elastic lamella or cartoid stenosis were produced. ${ }^{22)}$ Excessive pressure by clamp even leading to minimal damage must be avoided in operation. Our study histologically proved that the application of pressure control SMA clamp can prevent the vessel wall damage.

The clamping in surgical operation is required for the selection of suitable notch according to the vessel situation and diameters, mostly depending on the experience. ${ }^{23)}$ However, a previous study reported that vessel wall damage after clamping is not avoidable. ${ }^{24)}$ This may mean that the cause of the vessel wall damage is not only skillful or experiment of surgeons but also performance limitation of conventional clamp. We regarded the use of SMA properties, which is super elasticity, and applied its property to clamp. In this study, the damage score revealed no significant increase with different notch in SMA clamp, suggesting that SMA clamp can be used safety irrespective of skill or experiment of the surgeon by the regulation of pressure of SMA material.

This experiment confirmed that this unique mechanical property of SMA was effective in controlling clamp pressure. The new design successfully improved the clamps' safety. This pressure controlling method maybe highly valuable in many related research areas such as endoscope development and intravascular surgery.

\section{Acknowledgment}

The authors would like to thank Prof. Hideki Hosoda (precision and Intelligence Laboratory, Tokyo institute of technology) for assistance in the design of SMA wire, and Ms. Miho Oikawa and Ayako Okumoto (Graduate School of Biomedical Engineering, Tohoku University) for assistance in animal experiments and sample preparations. Funding for this work was received from Grants-inAid for Scientific Research (B) (no. 21390522, 20092011) from the Japan Society for the Promotion of Science.

\section{References}

1) Sachs M, Auth M, Encke A. Historical development of surgical instruments exemplified by hemostatic forceps. World J Surg 1998; 22: 499-504.

2) Luo Y, Kodaira S, Zhang Y, et al. The application of superelastic SMAs in less invasive haemostatic forceps. Smart Mater Struct 2007; 16: 1061-5.

3) Gersak B, Trobec R, Krisch I. et al. Loss of endothelium-mediated vascular relaxation as a response to various clamping pressures. Eur J Cardiothorac Surg 1996; 10: 684-9.

4) Darçin OT, Cengiz M, Ozardali I, et al. Pressure-controlled vascular clamp: a novel device for atraumatic vessel occlusion. Ann Vasc Surg 2004; 18: 254-6.

5) Margovsky AI, Lord RS, Meek AC, et al. Artery wall damage and platelet uptake from so-called atraumatic arterial clamps: an experimental study. Cardiovascular Surgery 1997; 5: 42-7.

6) Pabst TS3rd, Flanigan DP, Buchbinder D. Reduced intimal injury to canine arteries with controlled application of vessel loops. J Surg Res 1989; 47: 235-41.

7) Coelho JC, Sigel B, Flanigan DP, et al. Arteriographic and ultrasonic evaluation of vascular clamp injuries using an in vitro human experimental model. Surg Gynecol Obstet 1982; 155: 506-12.

8) Dujovny M, Wakenhut N, Kossovsky N, et al. Minimum vascular occlusive force. J Neurosurg 1979; 51: 662-8.

9) Margovsky AI, Chambers AJ, Lord RS. The effect of increasing clamping forces on endothelial and arterial wall damage: an experimental study in the sheep. Cardiovascular Surgery 1999; 7: 457-63.

10) Moore WM, Manship LL, Bunt TJ. Differential endothelial injury caused by vascular clamps and vessel loops. I. Normal vessels. Am Surg 1985; 51: 392-400.

11) Ozalp B, Canbaz S, Huseyinova G, et al. Histopathological comparison of vascular wall damage created by external cross-clamp and endoluminal balloon occlusion techniques. J Cardiovasc Surg 2009; 50: $545-53$.

12) Harvey JG, Gough MH. A comparison of the traumatic 
effects of vascular clamps. Br J Surg 1981; 68: 267-72.

13) Dogliotti AM, Guglielmini G. A new two-lever compression clamp for vascular surgery. Surgery $1955 ; \mathbf{3 8}$ : 717-9.

14) Vogel PM, Parise C. Comparison of SMART stent placement for arteriovenous graft salvage versus successful graft PTA. J Vasc Interv Radiol 2005; 16: 161926.

15) Es-Souni M, Es-Souni M, Fischer-Brandies H. Assessing the biocompatibility of NiTi shape memory alloys used for medical applications. Anal Bioanal Chem 2005; 381: 557-67.

16) Gall K, Tyber J, Brice V, et al. Tensile deformation of NiTi wires. J Biomed Mater Res A 2005; 75: 810-23.

17) Zhao S, Luo Y, Wang MH. Design of an SMA embedded clamp for endoscopic surgery. Int J Appl Electromagn Mech 2010; 33: 973-8.

18) Neville RF, Padberg FT, DeFouw D, et al. The arterial wall response to intimal injury in an experimental model. Ann Vasc Surg 1992; 6: 50-4.
19) Szczesny W, Cerkaska K, Tretyn A, et al. Etiology of inguinal hernia: ultrastructure of rectus sheath revisited. Hernia 2006; 10: 266-71.

20) Moore WM Jr, Bunt TJ, Hermann GD, et al. Assessment of transmural force during application of vascular occlusive devices. J Vasc Surg 1988; 8: $422-7$.

21) Gasser TC, Schulze-Bauer CA, Holzapfel GA. A three-dimensional finite element model for arterial clamping. J Biomech Eng 2002; 124: 355-63.

22) Dobrin PB, McGurrin JF, McNulty JA. Chronic histologic changes after vascular clamping are not associated with altered vascular mechanics. Ann Vasc Surg 1992; 6: 153-9.

23) Bhaskar J, Foo J, Sharma AK. Clamp-and-sew technique for traumatic injuries of the aorta: 20-year experience. Asian Cardiovasc Thorac Ann 2010; 18: 161-5.

24) Babin-Ebell J, Gimpel-Henning K, Sievers HH, et al. Influence of clamp duration and pressure on endothelial damage in aortic cross-clamping. Interact Cardiovasc Thorac Surg 2010; 10: 168-71. 